\title{
Evaluating the Outcomes of Surgical Versus Conservative Treatments in Head Injury: A Comparative Observational Study Using Different Scales
}

\author{
Sanisha Sunny ${ }^{1}$, Priyanka Vajjapally ${ }^{1}$, Sheshagiri Sharavana Bhava Bandaru', Venkateshwarlu

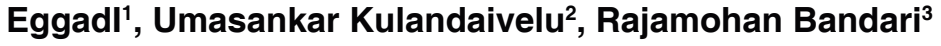

${ }^{1}$ Professor and Head,Department of Clinical Pharmacy,Vaagdevi College of Pharmacy ,Ramnagar, Hanamkonda, Warangal, Telangana, INDIA.

2Professor and Head, Department of Pharmaceutical Chemistry, KL College of Pharmacy, KLEF, Vaddeswaram, Guntur, Andhra Pradesh, INDIA.

${ }^{3}$ Neurosurgeon, MGM Hospital, Warangal, Telangana, INDIA.

\begin{abstract}
Background: This study is to evaluate the outcomes of surgical versus conservative treatment in head injury by using different scales to rate the quality of life in both the treatments. Aim: To evaluate the outcomes of surgical versus conservative treatment in Head injury. Methods: A prospective, comparative observational study was conducted in Neurosurgery department in a tertiary care teaching hospital for a period of 6 months. All the patients with head injury were included in the study and reviewed.Among the subjects two groups are made in which one group includes the subjects who are treated with conservative treatment and the other group who have undergone surgical treatment among these two groups GCS scale, four score scale and dementia rating scale are assessed and both the treatments are compared. Results: Patients with head injury between age groups of 20 to 70 years were recruited for this study. The patients recovery analysis according to GCS for conservative $(93.06 \%)$ and surgical $(50.40 \%)$, FSS for conservative $(90 \%)$ and surgical $(56.75 \%)$, DRS for conservative $(100 \%)$ and surgical $(50.04 \%)$. Conclusion: Based on the severity it is decided whether conservative or surgical treatment is given to the patient, but primary choice of treatment should be conservative treatment for patients with less severity as patients under conservative treatment had better recovery and memory compared to that of patients under surgical treatment.
\end{abstract}

Key words: Head injury, Glasgow scale, FOUR score scale, Dementia rating scale, Surgical, conservative.

\section{INTRODUCTION}

Head injury is a trauma to the scalp, skull or brain. It may be only a major or minor bump on the skull. ${ }^{1}$ Head injury may lead to bleeding in the brain tissues and in certain layers that surrounds the brain (subarachnoid haemorrhage, subdural haemorrhage and extradural haemorrhage). Head injury is one of the most common reasons for an emergency visit to the hospital. Traumatic brain injury (TBI) accounts for over 1 in 6 injury-related admissions each year. ${ }^{2}$ Traumatic brain injury is a leading cause of morbidity, mortality, disability, in India and other developing countries. Road traffic injuries are leading cause $(60 \%)$ of traumatic brain injury followed by falls (20-25\%), violence and alcohol involvement (15-20\%) in India. ${ }^{3,4}$ The occurrence of total traumatic brain injury has reminded similar throughout history in spite of modern Kevlar helmets. ${ }^{5}$ Head injuries are commonly caused by a blow to the head that are usually associated with vehicle accidents, falls and sports related accidents. The treatment of the condition depends on the seriousness of the injury. Mild traumatic brain injures requires overthe-counter pain relievers to treat headache and usually needs to be monitored closely at home for any persistent, exacerbating or new symptoms. Moderate to severe brain injuries
DOI: 10.5530/ijopp.13.2.20

Address for correspondence:

Prof. Venkateshwarlu Eggadi Professor and Head, Department of Clinical Pharmacy,Vaagdevi College of Pharmacy,Ramnagar, Hanamkonda, Warangal, Telangana, INDIA.

Phone no: +91-9848835092 Email Id: eggadivenkey@gmail. com

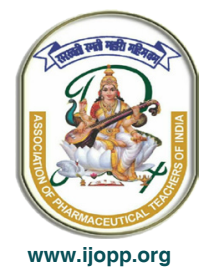


concentrate on enough oxygen, sufficient blood supply, blood pressure and avoid any further injury to the head. Treatment limitations for peripheral destruction of the brain immediately after an injury may contain: Diuretics, Anti-seizure drugs and Coma-inducing drugs. Urgent surgery is needed to reduce further damage to the brain. Surgery may be used for the following issues: Eliminate clotted blood (hematoma), repairing skull fractures, bleeding in the brain, opening in the skull. ${ }^{6}$ The Glasgow Coma Scale (GCS) is the most common scoring system used to describe the level of consciousness in a person following a traumatic brain injury. ${ }^{7}$ The full outline of unresponsiveness [FOUR] score is validated scale describing the essentials of a coma examination, including motor response, eye opening and eye movements, brainstem reflexes and respiratory pattern. ${ }^{8}$ The clinical Dementia Rating Scale is a numeric scale used to quantify severity of symptoms of dementia. Scores on each of these are combined to obtain a composite score ranging from 0 to $3 .^{9}$

\section{MATERIALS AND METHODS}

Study Design: A prospective comparative observational study.

Study Site: Mahatma Gandhi Memorial Hospital, Warangal, Telangana, India.

Study Period: Study was carried for 6 months duration July 2017 to January 2018, after approval from Ethics of Vaagdevi College of Pharmacy, India Protocol Number: KIEC/KMC/NCT/NIS/2018/P30.

Sample Size: 50 patients were considered in our study in which 25 patients were under conservative treatment and 25 patients were under surgical treatment in Department of Neurosurgery. The data was collected in Case Record Form.

Patient Inclusion Criteria: The patients between age groups of 10 to 70 years with head injury were included. They were divided into different groups.

Patient Exclusion Criteria: Patients less than 10 years, above 70 years, non-traumatic cases, long term treatment outcomes and associated injury to chest, abdomen and extremities were excluded.

Study Procedure: Parameter of efficacy and safety were evaluated by personal interaction with patient and recorded in Case Record Form. Efficacy parameter included changes in eye response, motor response, brainstem reflexes and memory loss in patients under conservative and surgical treatment. Safety profile was evaluated by recovery and adverse drug reactions observed in patients. First follow up was taken after 15 days and second follow up after one month of patient's treatment. Those patients who were receiving conservative and surgical treatment were interviewed personally after taking their written consent as the procedure is shown in Figure 1.

\section{Statistical analysis}

Data was analyzed by percentage calculation and unpaired t-test using GraphPad Prism version 8.

\section{RESULTS}

A total number of 62 patients have been enrolled in the study. Out of which 12 patients refused to co-operate with us. All the 50 patients who were included in the study were above 20 years of age was diagnosed with various types of head injuries using CT-scan.

Age Distribution: Among the involved patients according to age distribution 20-29 years (19 patients) were involved (38\%), 30-39 years (11 patients) were involved (22\%), 40-49 years (9 patients) were involved $(18 \%)$ and $50-59$ years (11 patients) were involved (22\%) was shown in Figure 2.

Gender Distribution: Figure 3 shows the males 34 (68\%) and females $16(32 \%)$ in head injury patients.

Types of Head Injury: According to types of head injuries 24 patients $(48 \%)$ showed mild type of head injury, 11 patients $(22 \%)$ showed moderate type of head injury and 15 patients $(30 \%)$ showed severe type of head injury that is shown in Figure 4.

GCS SCORE: In the present study 10 patients (35\%) showed GCS Score severe, 5 patients (17\%) showed GCS Score moderate and 14 patients (48\%) showed GCS Score mild in relation with different types of head injuries as shown in Figure 5.

FOUR SCORE: Among the patients in our study 9 showed severe effect according to FOUR score, 4 showed moderate effect according to FOUR score and 16 patients showed mild effect according to FOUR score in relation with different types of head injuries as shown in Figure 6.

DEMENTIA RATING SCALE: In our study 17 patients $(57 \%)$ showed DRS score 0, 1 patient (3\%) showed DRS score $0.5,1$ patient $(3 \%)$ showed DRS score 1, 2 patients (7\%) showed DRS score 2 and 9 patients $(30 \%)$ showed DRS score 3 in relation with different 
types of head injuries as shown in Figure 7.

\section{Comparison of Conservative Vs Surgical Treatment Based on GCS scale}

The total number of patients involved with different type of head injury is 50 in which 25 patients received conservative treatment shown $93.06 \%$ recovery and 25 patients underwent surgical treatment shown 50.40\% recovery. In present study conservative treatment patients

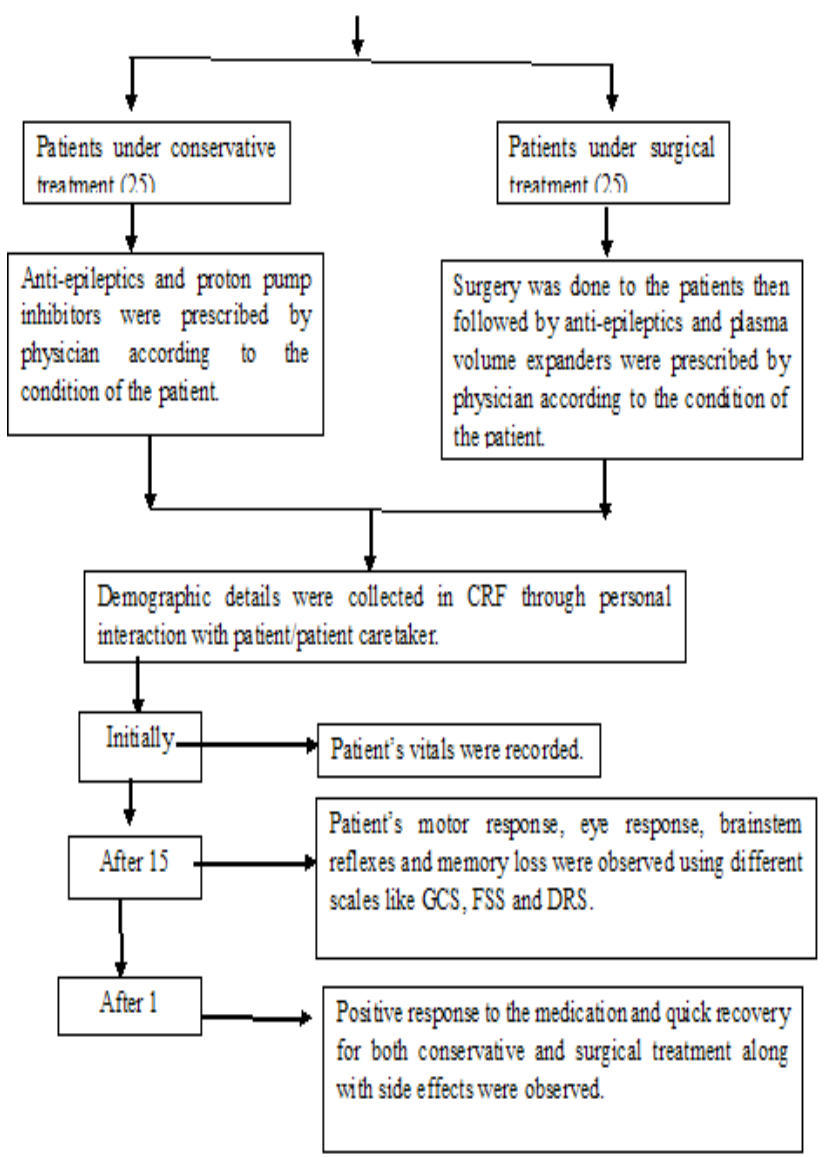

Figure 1:Consent of the study.

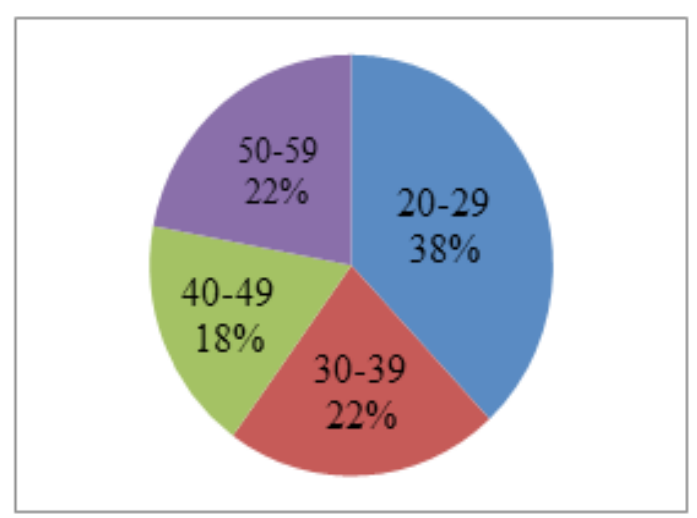

Figure 2: Total number of patients with age distribution. shows significantly $(p<0.00001)$ improved than surgical treatment. Comparison of conservative treatment versus surgical treatment in relation with GCS score based on prospective study is shown in Figure 8.

\section{Comparison of Conservative Vs Surgical Treatment Based on FOUR Score Scale}

The total number of patients involved with different types of head injury is 50 in which 25 patients received

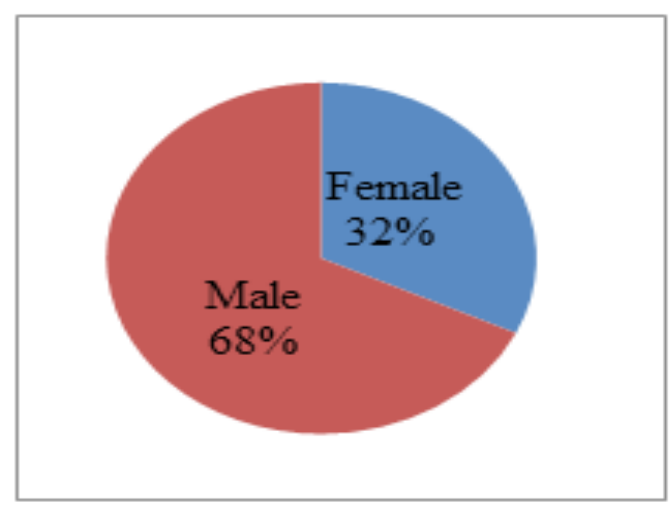

Figure 3: Total no of patients with gender distribution.

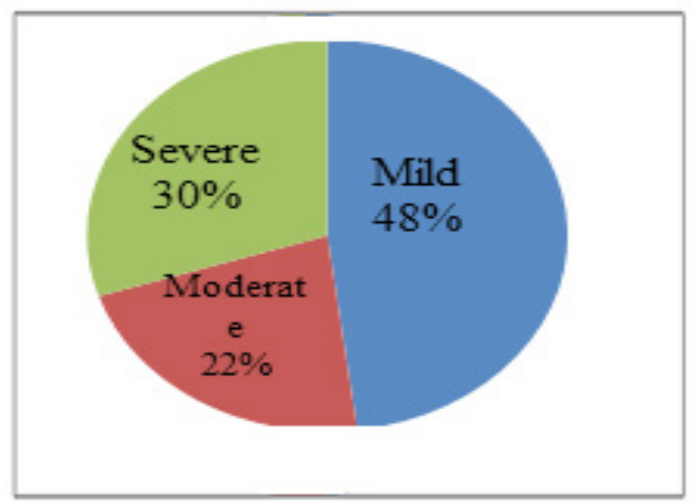

Figure 4: Total no of patients with types of head injuries.

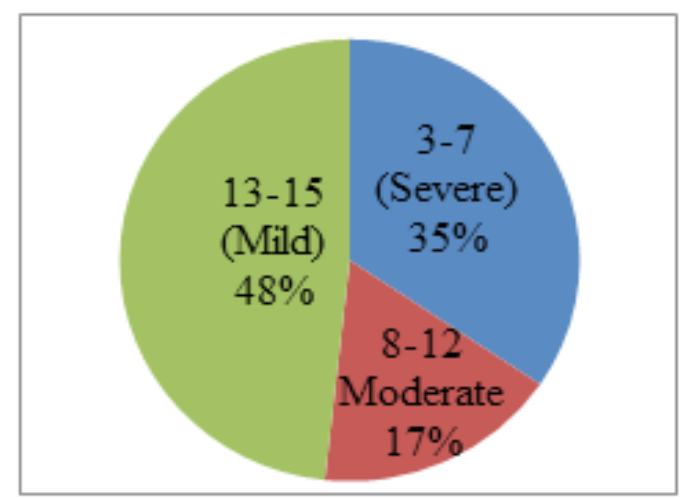

Figure 5: Total no of patients with different types of head injuries in relation with GCS scale.

Indian Journal of Pharmacy Practice, Vol 13, Issue 2, Apr-Jun, 2020 
conservative treatment has shown 90\% recovery and 25 patients has undergone surgical treatment has shown $56.75 \%$ recovery. In present study conservative treatment patients shows significantly $(p<0.00001)$ improved than surgical treatment. Comparison of conservative versus surgical treatment in relation with FOUR score based on prospective studies is as shown in Figure 9.

\section{Comparison of Conservative Vs Surgical Treatment Based on Dementia Rating Scale}

The total number of patients involved with different types of head injury is 50 in which 25 patients received conservative treatment has shown 100\% recovery and 25 patients who undergone surgical treatment has shown $50.04 \%$ recovery. Comparison of conservative versus surgical treatment in relation with Dementia rating scale based on prospective studies is as shown in Figure 10.

\section{DISCUSSION}

In the current study, total 50 patients diagnosed with Head injury were enrolled. Conservative or surgical treatments were prescribed by physician according to patient's suitability. Traumatic brain injury (TBI) is followed by many changes in cognition, social interaction,

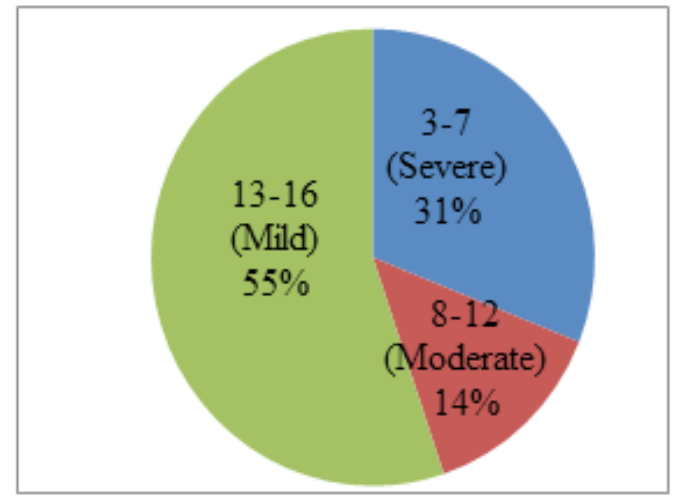

Figure 6: No of patients with different types of head injuries in relation with FOUR score.

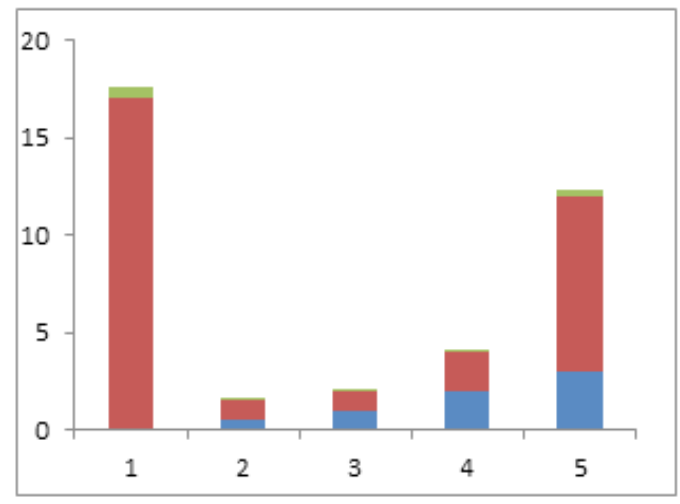

Figure 7: Total no of patients with gender distribution. functioning and communication. ${ }^{10}$ The outcome of the study revealed that 20-60 is the age of prevalence in TBI which was an assistance to study done by Ross Zafonate et al. who reported 18-70 is the age of prevalence in TBI. ${ }^{11}$ Study proved that the current distribution of severe, moderate and mild TBI as 29\%, 4\% and $67 \%$ respectively ${ }^{11}$ which was a support to our study, where we have current distribution of severe, moderate and mild as 30\%,2\%, 48\% respectively. Subjects enrolled in the study were $68 \%$ of males and $32 \%$ of females which

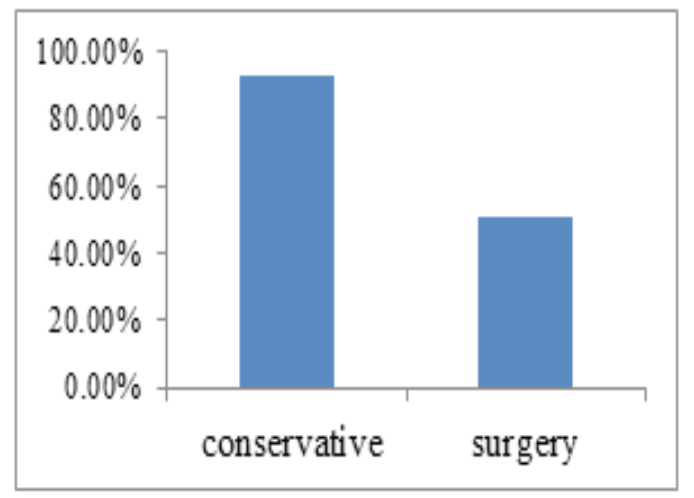

Figure 8: Comparison of total no of patients with conservative and surgical treatment using GCS score.

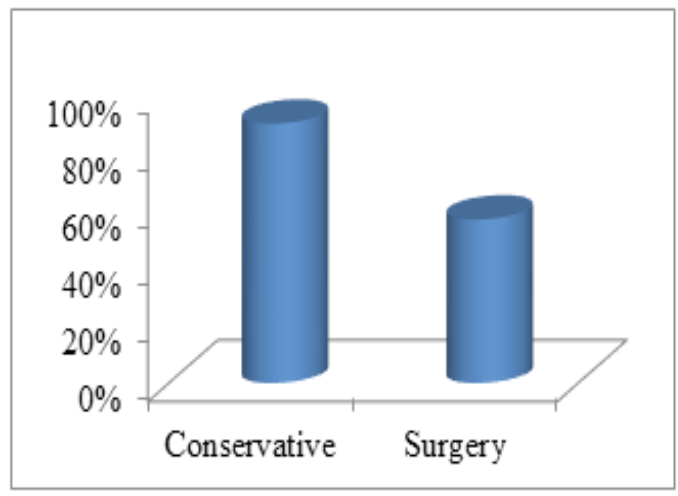

Figure 9: Comparison of total number of patients with conservative and surgical treatment using FOUR score scale.

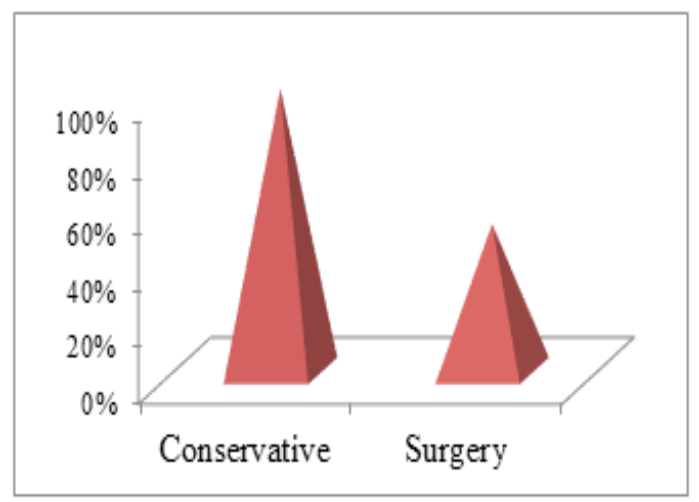

Figure 10: Comparison of total number of patient's conservative treatment with surgical treatment using Dementia rating scale. 
matched the criteria of the study done by Hanish et al. ${ }^{12}$ who reported $77.1 \%$ of males and $22.9 \%$ of females. ${ }^{12}$

Ross et al. had correlated the GCS score with TBI patients and found that $80.5 \%$ had GCS score (13-15), $10.5 \%$ had GCS score (9-12) and 9\% had GCS score (3-7), ${ }^{11}$ which was complementary to the study. Where we concluded $48 \%$ had GCS score (13-15), 17\% had GCS score (9-12) and $34 \%$ had GCS score (3-7). Blenow et al. disclosed information on FOUR scale score with TBI patients and found $62.86 \%$ had score (13-15), $10.67 \%$ had score (9-12) and $26.47 \%$ had score $(<8)$ which correlated the study proved that $55 \%$ had score (13-16), 14\% had (9-12) and $31 \%$ had score $(<8) .{ }^{8}$ In our study the outcome of CDR scale score demonstrated 17 patients with no memory loss with score 0,1 patient with very mild dementia with score $0.5,1$ patient with mild dementia with score 1,2 patients with moderate dementia with score 2 and 9 patients with severe dementia with score 3 .

In this study the comparison of conservative versus surgical treatment using GCS score showed that the patients under conservative treatment are showing improved positive response of $(93.06 \%)$ compared to patients under surgical treatment showing positive response of $(50.40 \%)$. In this study the comparison of comparative versus surgical treatment using FOUR score scale showed that the patients under conservative treatment are showing improved positive response of $(90 \%)$ compared to patients under surgical treatment showing positive response of $(56.75 \%)$. And the study comparison of conservative versus surgical treatment using Dementia rating scale showed that the patients under conservative treatment are showing improved positive response of $(100 \%)$ compared to patients under surgical treatment showing positive response of $(50.04 \%)$.

Regardless of all the efforts, there were some hindrance of the study like small sample size and short duration (6 months) due to this adequate sample size was not resulted. Patients were not coming for systematic follow ups. Therefore, few patients were contacted telephonically and heavy flow of OPD patients limits the pharmacist and patient's interaction.

\section{CONCLUSION}

This prospective, observational study "Comparison of Conservative versus Surgical treatment in head injury: Based on different scales" was done in a safe manner both in males and females. According to our study patients under conservative treatment were showing positive response to the medications and quick recovery when checked with GCS scale, FOUR score and
Dementia rating scale and reported mild side effects such as headache and vertigo compared to those patients under surgical treatment reported mostly with death of the patients mainly due to surgical infections, DVT, bleeding and atelectasis. But in certain conditions like clot formations, skull fractures, aneurysm, brain abscess and bleeding in brain we have to go with surgical treatment to overcome life threatening conditions. Based on the severity it is decided wheather conservative or surgical treatment is given to the patient, but primary choice of treatment should be conservative treatment for patients with less severity as there is less changes of complete recovery for patients with surgical treatment.

\section{ACKNOWLEDGEMENT}

I would like to thank my parents and friends Mr.Alan M Jojo, Mr. S. Nikhil Reddy, Miss. Sarika for their support in completion of the project.

\section{CONFLICT OF INTREST}

The authors declare no conflict of interest.

\section{ABBREVIATIONS}

TBI: Traumatic Brain Injury, FSS: Four Score Scale, GCS: Glasgow Coma Scale, DRS: Dementia Rating Scale, FOUR: Full Outline Of Unresponsiveness.

\section{SUMMARY}

Conservative and Surgical treatments are the available options in head injury. Based on the severity and treatment outcomes, conservative treatment is also efficacious and has less burden in treating head injury patients with better out comes. It depends on the severity of the patient case by case to choose surgery or conservative modalities of treatment.

\section{REFERENCES}

1. Liebig CW, Congeni JA. Sports-related traumatic brain injury (concussion). Nelson Textbook of Pediatrics. 20 $0^{\text {th }}$ ed. Philadelphia, PA: Elsevier. 2016;688.

2. Papa L, Goldberg SA. Head trauma. Rosen's Emergency Medicine: Concepts and Clinical Practice. 9th ed. Philadelphia, PA: Elsevier. 2018;34.

3. Gururaj G. Epidemiology of traumatic brain injuries: Indian scenario. Neurological Research. 2002;24(1):24-8.

4. Marshall LF. Head injury: Recent past, present and future. Neurosurgery. 2000;47(3):546-61.

5. Mah K, Hickling A, Reed N. Perceptions of mild traumatic brain injury in adults: A scoping review. Disability and Rehabilitation. 2017;1-15.

6. Snell DL, Siegert RJ, Hay-Smith EJ, et al. An examination of the factor structure of the revised illness perception questionnaire modified for adults with mild traumatic brain injury. Brain Injury. 2010;(13-14):1595-605.

7. Teasdale G, Allen D, Brennan P, McElhinney E, Mackinnon L. The Glasgow Coma Scale: An update after 40 years. Nursing Times. 2014;110:12-6.

Indian Journal of Pharmacy Practice, Vol 13, Issue 2, Apr-Jun, 2020 
8. Blennow K, Hardy J, Zetterberg H. The neuropathology and neurobiology of traumatic brain injury. Neuron. 2012;76(5):886-99.

9. Hughes CP, Berg L, Danziger W, Coben LA, Martin RL. A new clinical scale for the staging of dementia. The British Journal of Psychiatry. 1982;140(6):566-72.

10. Stratton MC, Gregory RJ. After traumatic brain injury: A discussion of consequences. Brain Injury. 1994;8(7):631-45.
11. Zafonte R, Friedewald WT, Lee SM, Levin B, Diaz-Arrastia R, Ansel B, et al. The citicoline brain injury treatment (COBRIT) trial: Design and methods. Journal of Neurotrauma. 2009;26(12):2207-16.

12. Bansal H, Chaudhary A, Singh A, Paul B, Garg R. Decompressivecraniectomy in malignant middle cerebral artery infarct: An institutional experience. Asian Journal of Neurosurgery. 2015;10(3):203-6. 